\title{
Millimetre Wave Communication with Spatial Division Multiplexing for 5G systems
}

\author{
Keshav N, Kate Akshay Vijay, Ravi Kumar CV, Kalapraveen Bagadi \\ School of Electronics Engineering, VIT University, Vellore, India
}

Article History: Received: 11 January 2021; Revised: 12 February 2021; Accepted: 27 March 2021; Published online: 16 April 2021

\begin{abstract}
The point of this paper is to combination of spatial division multiplexing (SDMA) along with NOMA improves the performance of the system. This paper is done with multibeam forming for performance enhancement, Analog beam forming is done along with the digital beam forming to increase the spectral efficiency and also the user count increases so that we could able to achieve high throughput. In this paper the design problems of mm wave NOMA due to beamforming is focused so that achievable data rate is high. This is due to the number of RF chains. We analyses the demanding joint plan of the intertwined power distribution and client matching for mm Wave-SDMA. Some problems are talked about and a few arrangements are proposed in detail in mm Wave communications.
\end{abstract}

Keywords: millimeter wave (mm wave), non-symmetrical different access(NOMA), mm wave NOMA, beam forming.

\section{Introduction}

Throughout the years various get to strategies have picked up prominence and are being utilized as a part of extensive variety of continuous applications. There are numerous various get to methods like Frequency Division Multiple access (FDMA), isolated into little recurrence channels and distinctive channels are dispensed to various clients - like in Frequency Modulation (FM) radio. Numerous clients can transmit in the meantime however on various channels. Time division multiple access (TDMA), in which numerous clients can send information in various vacancies in same channel. Code division multiple access (CDMA), in which Clients may transmit in the meantime utilizing a similar recurrence band yet utilizing diverse codes so we can interpret to recognize a specific client. We regularly utilize a blend of FDMA+TDMA to accomplish a more prominent number of different channels as clarified underneath: Transmission utilizing distinctive Frequency bands in the meantime equal to two channels. Likewise, Transmission utilizing distinctive schedule openings yet same frequency band equal to two channels. Be that as it may, transmission utilizing two Frequency bands and two schedule openings equal to four channels.

Millimeter waves consisting of different frequencies that varies from 30GHZ to 300GHZ. They're found in the range between microwaves $(1 \mathrm{GHz}$ to $30 \mathrm{GHz}$ ) and infrared (IR) waves, which is known as incredibly high recurrence (EHF)at times.

In $\mathrm{mm}$ Wave interchanges, enormous staged clusters are generally received to defeat the high propagation misfortune, through $\mathrm{mm}$ Wave beam forming, which alludes to the simple or half breed beamforming. Both simple beam forming and cross breed beam forming are dependent upon the steady modulus (CM) limitation on using just stage shifters to control the reception apparatus loads. In NOMA, power designation between various NOMA clients and client matching are done to improve the presentation of the system. When joining mm Wave interchanges and NOMA, mm Wave beam forming isn't just for expanding shaft pick up, yet additionally increases the user count for multiple non-orthogonal multiple access users. These applications include, radio stargazing, far off detecting, car radars, military applications, imaging. Security screening, and media communications.

\section{I.SYSTEM MODEL}

The schematic diagram given below figure 1 is everything based on full associated stage shifter based pattern. The half breed patterns having around M RF chains subsequently it uphold all things considered M clients by utilizing SDMA. Various clients presently can be served in a similar RB with simple beam forming. Due to the decreased channel gains, achievable rate of the user decreases as compared to the NOMA users, because of these issues NOMA users count is limited commonly. The important strategy to build the complete number of mm wave-NOMA clients is to utilize half and half SDMA and NOMA.Specifically the Bs has half breed beam forming pattern around M RF chains, and adventure NOMA with every RF chain and SDMA between various RF chains. On the off chance that every RF chain is able to provide service toK NOMA clients, the mixture SDMA and NOMA methodology can uphold all things considered MK clients.

In this figure 2 the mmwave NOMA base station consist simple beam forming provided with 2 clients. Between Users 2 and 3, the BS just necessities to frame a limited pillar, on the grounds that the point hole middle of User 2 and 3 is little. In such a case, the pillar gain is very huge. Notwithstanding, Between Users 1 
and 4, the BS needs to frame a lot more extensive bar, on the grounds that the point hole between Users 1 and 4 is a lot bigger. Accordingly, the bar gain of the BS is very less, which debases the presentation of mm WaveNOMA. here we get $2 \mathrm{RF}$ chains in each and everyone underpins into 2 NOMA clients first one is called setup model. The BS sets beam forming vector of the primary RF chain, i.e., RF 1, to frame two limited pillars to cover Users 4 and 1, having a place with a NOMA gathering. In this cycle, multi-pillar framing is received. In the interim, the Base station provides beam forming vectors of the subsequent RF chain, i.e., RF 2, to shape in addition to this we have two tight pillars to cover Users 2 and 3, having a place with another NOMA gathering. Force designation is performed among just the clients of a similar gathering.

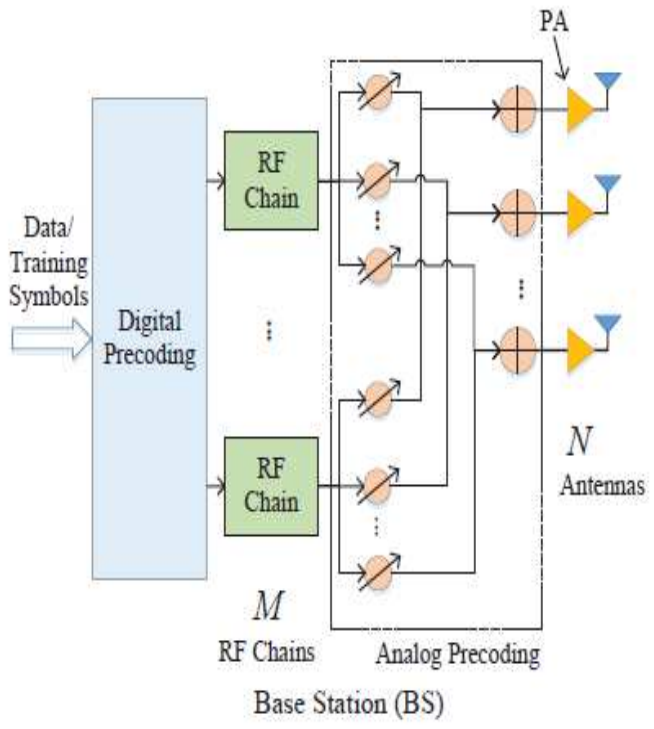

Fig. 1 Hybrid Analog/Digital beam forming

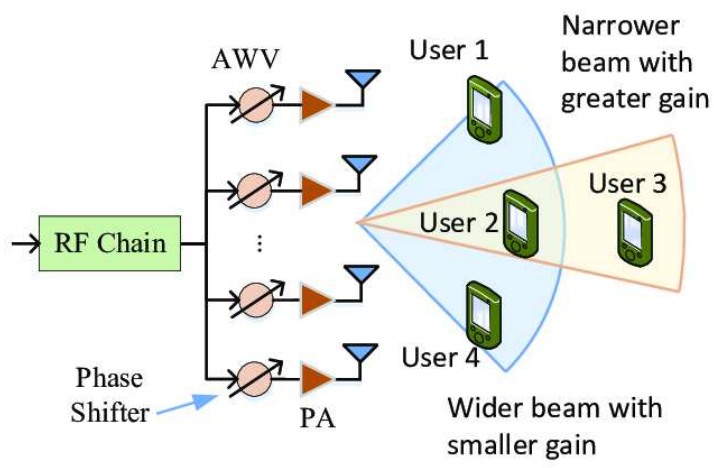

Base Station (BS)

Fig. 2 A mm-wave NOMA with simple beam forming at the base station

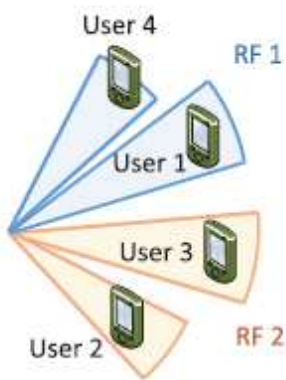

Mode 1

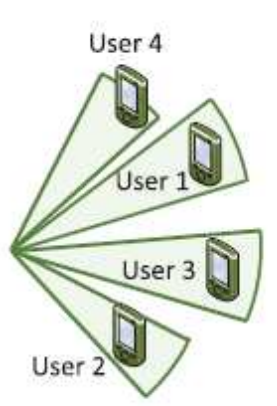

Mode 2

Fig. 3 NOMA mode interchange 


\section{II.Beam Forming}

The traditional one is a technique that focuses a wireless signal towards receiving end, rather than sending in all directions.

\subsection{Single beam forming mm-wave noma}

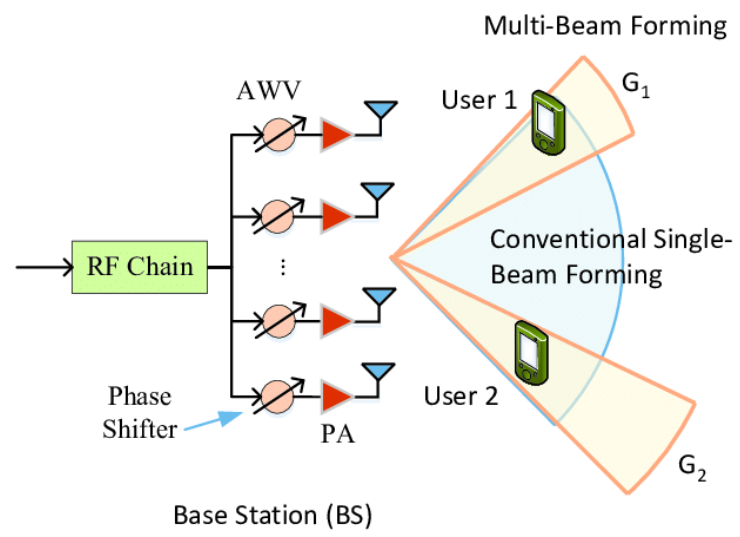

Fig. 4 single beam forming mm-wave noma

Beam forming is a ground-breaking method sometimes generally employed in sign preparing, radar and sonar, biomedical, and mainly in interchanges. In utilizations of beam forming in interchanges frameworks, the basic idea has to ideally deal with signals got over various radio wires, or the signs it is communicated over various ways, by changing the sign amplitudes and stages, to shape a solid pillar toward the bearing of interest, and simultaneously, to abstain from getting or making impedance.

2.2Multiple beam forming mm-wave noma

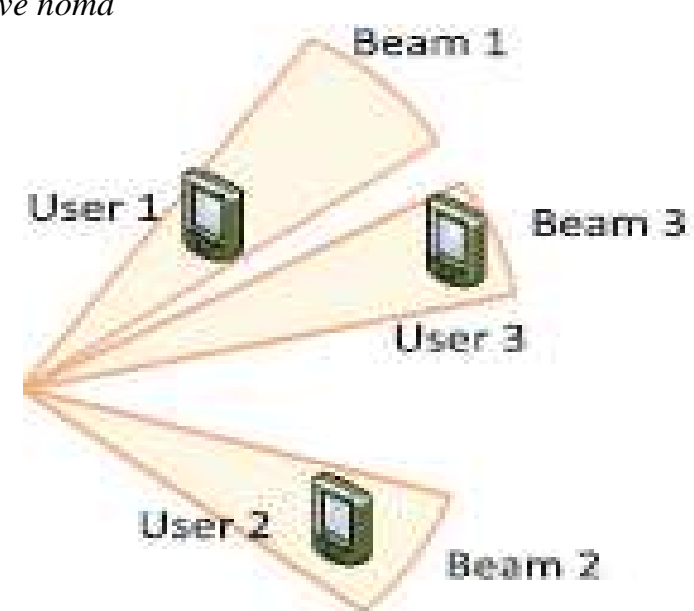

Fig. 5 Multi beam forming mm-wave noma

III.Simulation Analysis

Mock-up results obtained by correlarion of the total rate execution between various beam forming strategies are outlined in figure 5 . 


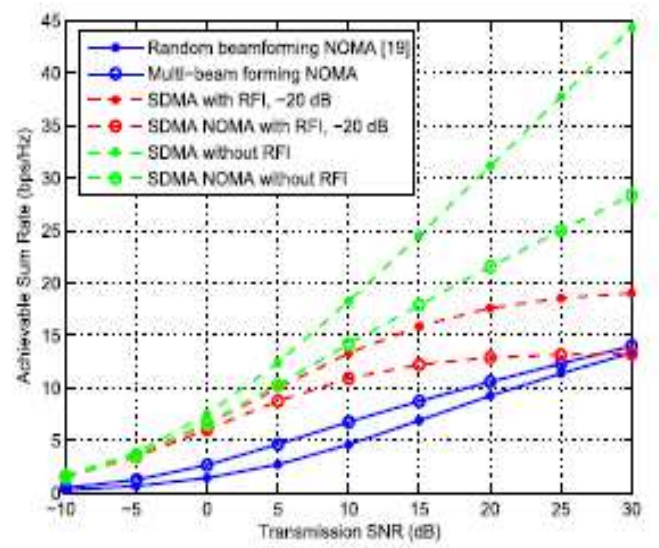

Fig. 6 Various beam forming tech.

In this figure 6:400 samples are taken as input and super position modulated-4 bit QAM is done for converting the samples into 16 symbols (four rows-4 symbols in each row) for transmission. Real and imaginary part of gain matrix is shown in the figure 7.Yellow color of the imaginary part shows the enlarged bandwidth.

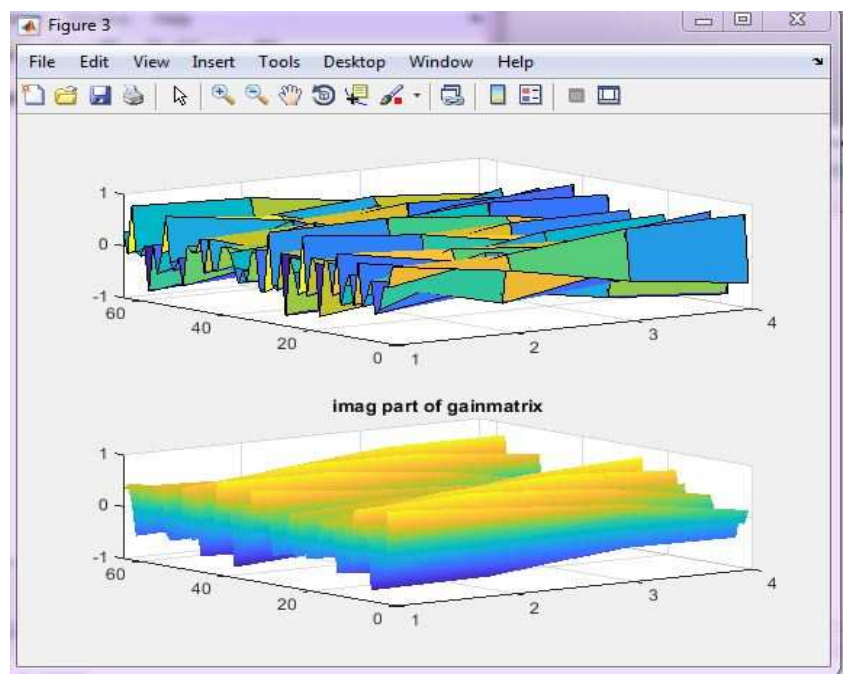

Fig 7 Various beam forming tech data signal

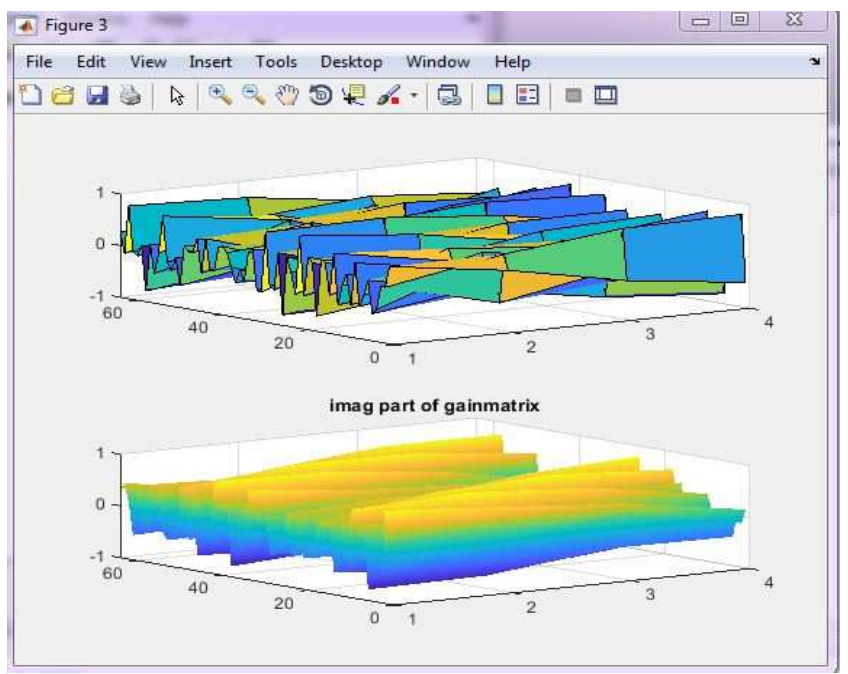

Fig. 8 Various beam forming tech for gain matrix 


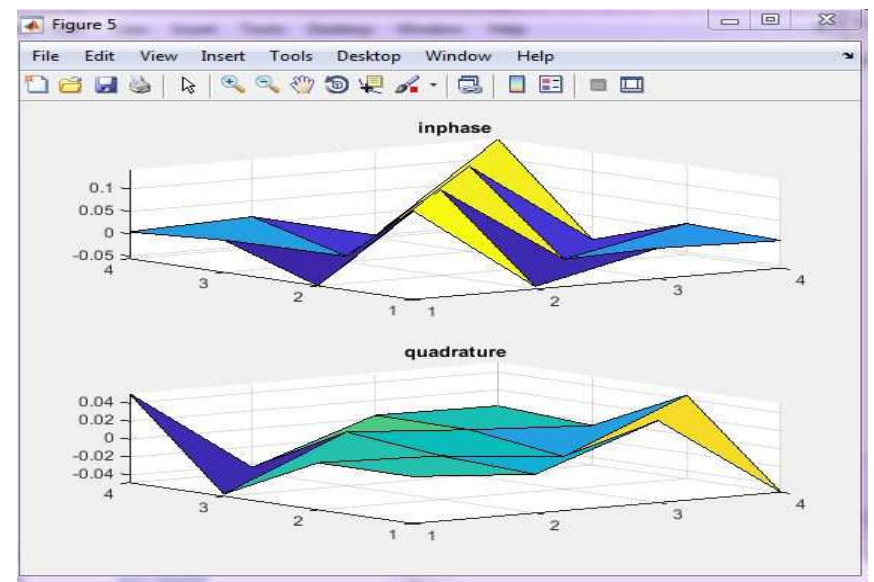

Fig.9 Various beam forming for phase of the signal

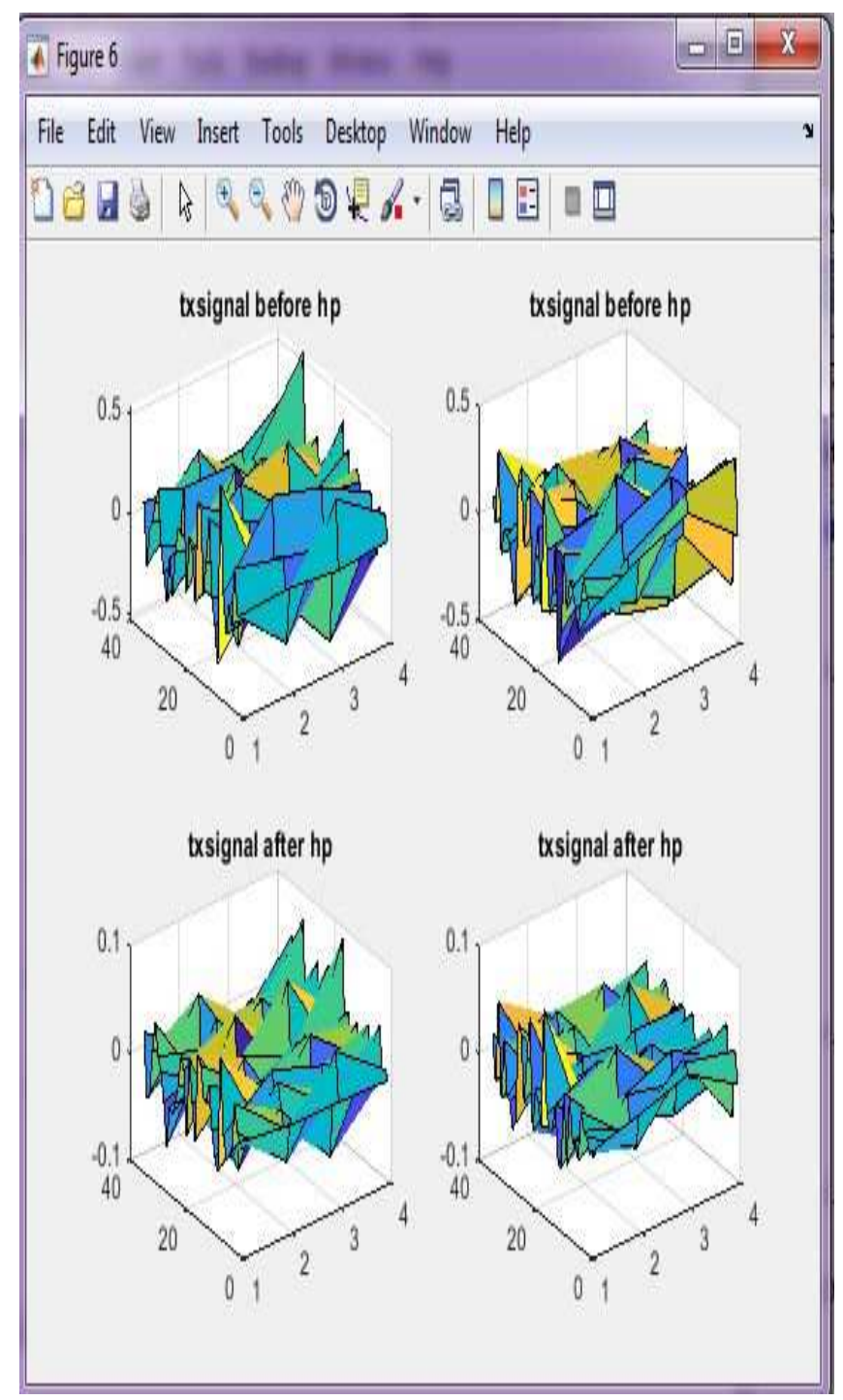

Fig.10 Hybrid precoding of the transmitted signal 


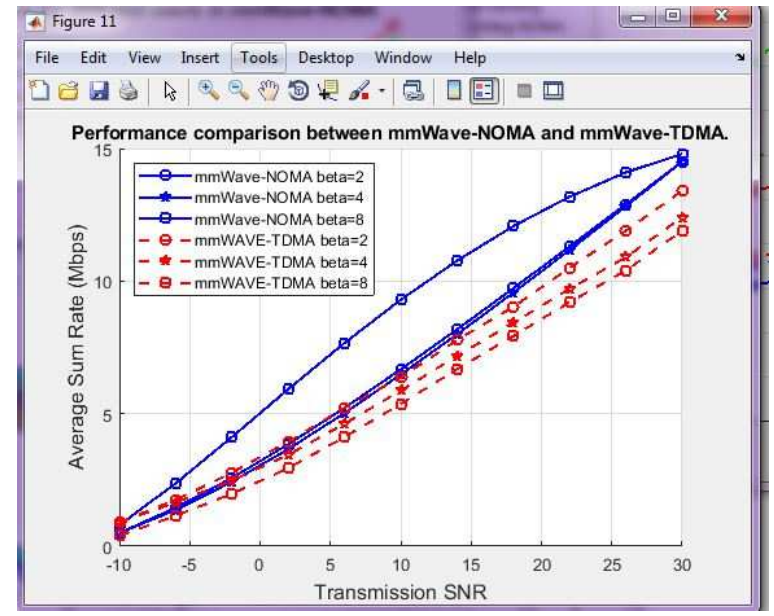

Fig.11 Hybrid precoding of the transmitted signal

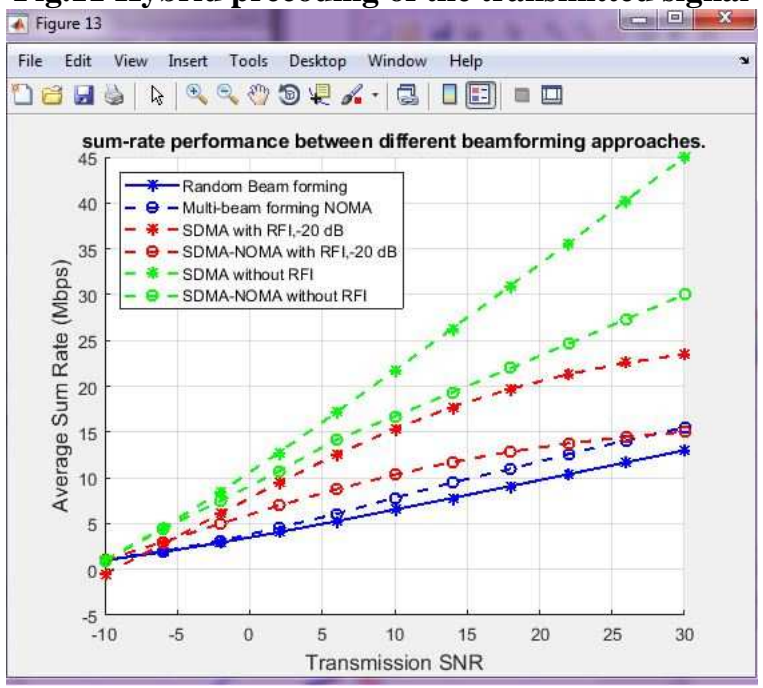

Fig.12 Hybrid precoding of the transmitted signal with NOMA and SDMA

\section{Conclusions}

This paper mainly focused $5 \mathrm{G}$ techniques but we are facing several issues in beam forming of mmwaveNOMA.in our paper we have shown both analog and digital precoding is done at the transmitter side. Analog and digital combiner are used at the receiver side.AOA(Arrival of Azimuth angle) and AOD(departure of Azimuth angle) are done along with NOMA.We compared both conventional single beam forming and multibeam forming for mm wave NOMA where NOMA can accomplish improved entirety rate execution and vigor. High efficiency,throughput and data rate is achieved and mainly the user count is increased due to these methods. examinations. From the broad reenactment think about, it is found that, the traditional beneficiaries result high mistake floor as they can't alleviate arbitrary plentifulness execution, its many-sided quality increments exponentially with number of clients and tweak arrange. As we can see the mm wave noma with different beta values at SNR equal to $15 \mathrm{db}$ sum rate of $12 \mathrm{Mbps}$.

\section{REFERENCES}

[1] R. Prasad, OFDM for wireless communications systems, Vol. 1,Artech House,London,

[2] S. B. Weinstein, and P. M. Ebert, "Data transmission by frequency-division multiplexing using the discrete Fourier transform" IEEE Transactions on Communications, Vol. 19, No.5, pp. 628-634, 1971.

[3] L. Miller, and J. Lee, CDMA Systems Engineering Handbook, Vol. 1, Artech House, London,

[4] A. J. Viterbi,CDMA: Principles of Spread Spectrum Communication, Vol. 2, Addison-Wesley, Boston, 1995.

[5] L. Miller, and J. Lee,CDMA Systems Engineering Handbook, Vol. 1, Artech House, London, 1998. 
[6] Y. Yan, and M. Ma, "Novel frequency-domain oversampling receiver for CP MC-CDMA systems", IEEE Communications Letters, Vol. 19, No. 4,pp. 661-664, 2015.

[7] R. Prasad, and S. Hara, "Overview of multicarrier CDMA”, IEEE Communications Magazine, Vol. 35, No. 12, pp. 126-133, 1997.

[8] A. C. McCormick, and E. A. Al-Susa, "Multicarrier CDMA for future generation mobile communication”, Electronics \& communication Engineering Journal, Vol. 14, No. 2, pp. 52-60, 2002.

[9] G. Fettweis, A. S. Bahai, and K. Anvari, "On multi-carrier code division multiple access (MC-CDMA) modem design”, In: Proc. of IEEE Vehicular Technology Conf., Stockholm, pp. 1670 -1674,1994.

[10] L. Hanzo, and T. Keller,OFDM and MC-CDMA: A primer, Vol. 2, John Wiley, West Sussex, 2006.

[11] N. Yee, and J. P. Linnartz, "Multicarrier CDMA in indoor wireless radio networks", In:Proc. of PIMRC, Yokohama, pp. 109-113, 1993.

[12] J. G. Proakis,Digital Communications, Vol. 4, Mc-Graw Hill, New York, 1995.

[13] Emarah, A. M., Metwally, K. G., \& Zaghw, A. I. Comparative Analytical Study of Reinforced Concrete Wall Subjected to Blast Loading Pattern. BEST: International Journal of Management Information Technology and Engineering (BEST: IJMITE), 5(09), 73-78.

[14] Nuno S, dinnis R. Reliability of an IB-DFE in the presence of Channel Estimation Errors. Proceedings of the 77th IEEE VTC 2013, 1-5.

[15] Dinis R, Silva P, Gusmao A. An iterative frequency-domain decision-feedback receiver for MCCDMA schemes. Proceedings of the 61st IEEE VTC 2005, 271-275S. Verdu, Multiuser Detection, Vol. 1, Cambridge University Press, Cambridge, 1998.

[16] V. Bhaskar, and L. S. Pai, "Performance analysis of MC-CDMA systems under Nakagami Hoyt Fading”, Wireless Personal Communications, Vol. 69, No. 4, pp. 1885-1898, 2013.

[17] A. Silva, S. Teodoro, R. Dinis, and A. Gameiro, "Iterative frequency-domain detection for IA-precoded MC-CDMA system", IEEE Transactions on Communications, Vol. 62, No. 4, pp. 1240-1248, 2014.

[18] Y. Yan, and M. Ma, "Novel frequency-domain oversampling receiver for CP MC-CDMA systems", IEEE Communications Letters, Vol. 19, No. 4,pp. 661-664, 2015.

[19] MANOGNA, T., \& KUMAR, M. S. DESIGN OF HORN ANTENNA ARRAYSFOR THE GENERATION OF LOW SIDELOBES.

[20] W. L. Sung, Y. K. Chang, F. B. Ueng, and Y. S. Shen, "A New SAGE-Based Receiver for MC-CDMA Communication Systems", Wireless Personal Communications, Vol. 85, No. 3, pp. 1617-1634, 2015.

[21] S. Haykin, Neural networks, Vol. 2, Pearson Education, Singapore, 1999

[22] K. Hornik, "Multilayer feedforward networks are universal approximators",Neural Networks, Vol. 2, No. 5, pp. 359-366, 1989.

[23] K. Hornik, "Approximation capabilities of multilayer feedforward networks", Neural Networks, Vol. 4, No. 2,pp. 251-257, 1991.

[24] N. Taspnar, and M. Cicek, "Neural Network Based Receiver for Multiuser Detection in MC-CDMA Systems", Wireless Personal Communications, Vol. 68, No. 2, pp. 463-472, 2013.

[25] Do, T. M. D., Lam, T., Ngo, V. T., \& Nguyen, T. T. N. (2020). Analysis of high performance concrete with steel fiber reinforcement. IJMPERD, 10(3), 8139-8147.

[26] K. P. Bagadi, and S. Das, "Efficient complex radial basis function model for multiuser detection in a space division multiple access/multiple-input multiple-output-orthogonal frequency division multiplexing system”, IET Communications, Vol. 7, No. 13, pp. 1394-1404, 2013.

[27] K. P. Bagadi, and S. Das. "Neural network based multiuser detection techniques in SDMA-OFDM system”, In:Proc. of Annual IEEE India Conf., Hyderabad, pp. 1-4, 2011.

[28] K. P. Bagadi, and S. Das. "Neural network-based multiuser detection for SDMA-OFDM system over IEEE $802.11 \mathrm{n}$ indoor wireless local area network channel models", International Journal of Electronics, Vol. 100, No. 10, pp. 1332-1347, 2013.

[29] J. C. Patra, R. N. Pal, R. Baliarsingh, and G. Panda, "Nonlinear Channel Equalization for QAM Signal Constellation Using Artificial Neural Networks", IEEE Transactions on Systems, Man, and Cybernetics, Vol. 29, No. 2, pp. $262-271,1999$.

[30] VARAPRASAD, S. P., \& RAO, R. P. DESIGN AND ANALYSIS OF A MULTI LAYER SUBSTRATE SINGLE PATCH MICROSTRIP PATCH ANTENNA FOR ENHANCING THE BEAM WIDTH WITH CONTROL ON DIRECTIVITY.

[31] K. P. Bagadi, and S. Das, "Multiuser Detection in SDMA-OFDM Wireless Communication System Using Complex Multilayer Perceptron Neural Network", Wireless personal communications, Vol. 77, No. 1, pp. 21-39, 2014.

[32] K. P. Bagadi, and S. Das, "Neural network-based adaptive multiuser detection schemes in SDMAOFDM system for wireless application", Neural Computing and Applications, Vol. 23, No. 3, pp. 1071-1082, 2013. 
[33] Modeling and Analyzing Millimeter Wave Cellular SystemsIEEE transactions on communications, Jeffrey G. Andrews, Fellow, Tianyang Bai, Student Member, Mandar N. Kulkarni, Student Member, Ahmed Alkhateeb, Student Member, Abhishek K. Gupta, and Robert W. Heath, Jr., Fellow,vol. 65, no. 1, january 2017. [34] AGRAWAL, E., \& MANU, K. INTERCHIP INTERCONNECTIONS.

[35] C.V. Ravikumar, Saranya KC," Implementing Mobile adhoc Networks with improved AODV protocol" International Journal of Applied Engineering and Research,2016, Vol 11,No. 9, pp. 6284-6289

[36] C.V. Ravikumar, Kala Praveen Bagadi," Receiver design using artificial Neural Network for signal detection in MC-CDMA system." International Journal of Intelligent Engineering \& Systems”,2017 Jan

[37] C.V. Ravikumar, Kala Praveen Bagadi, "Performance analysis of HSRP in provisioning Layer-3 Gateway Redundancy for Corporate Networks." Indian Journalof Science and Technology,2016 may ,Vol. 9 issue 20.89851 .

[38] Do, T. M. D., Lam, T. Q. K., \& Ngo, V. T. (2020). Analysis of textile and steel fibers based reinforced concrete beam. IJMPERD, 10(3), 8033-8040.

[39] C.V. Ravikumar, Kala Praveen Bagadi," Robust Neural network based multiuser detector in MCCDMA mMAI mitigation.” Indian Journalof Science and Technology,2016 Aug, Vol. 9 issue 30 .95994.

[40] C.V. Ravikumar, Kala Praveen Bagadi," Receiver design using ANN for signal detection in MCsystem.” International journal of Intelligent Engineering and systems,2017 Apr,

J. Clerk Maxwell, A Treatise on Electricity and Magnetism, 3rd ed., vol. 2. Oxford: Clarendon, 1892, pp.68-73. 